\title{
The Dalila effect: C57BL6 mice barber whiskers by plucking
}

\author{
Justyna R. Sarna, Richard H. Dyck, Ian Q. Whishaw * \\ Department of Psychology and Neuroscience, University of Lethbridge, and NeuroDetective Inc., Lethbridge, Alta, Canada, T1K 3M4
}

Received 28 May 1999; received in revised form 5 August 1999; accepted 9 August 1999

\begin{abstract}
Group-housed laboratory mice are frequently found with their whiskers and facial hair removed. It has been proposed that dominant mice are responsible for barbering the hair of the recipient (the Dalila effect), and early studies suggest that the hair is removed by nibbling. In the present study, pairs of C57BL6 mice, composed of a barber and recipient, were separated to allow hair to regrow. The animals were then placed together in an observation box and their social behavior was videorecorded. The videorecording was subjected to frame-by-frame analysis. Barbering was found to occur during acts of mutual grooming. During grooming, one member of a mouse pair removed the vibrissae of the conspecific and did so by grasping individual whiskers with the incisors and plucking them out. Although plucking appeared 'painful', recipients were passive in accepting barbering, and even pursued conspecifics for further grooming. Other measures indicated that barbers were heavier than recipients and brain weights were not different. Although cortical barrel fields appeared normal to cytochrome oxidization and zinc staining, Golgi analysis of layer three, barrel-field basilar dendrites indicated changes in cell morphology. The results are discussed in relation to the hypothesis that barbering is an expression of social dominance, the origins of the barbering behavior, and the consequences of barbering on brain function. (C) 2000 Elsevier Science B.V. All rights reserved.
\end{abstract}

Keywords: Aggressive behavior; Barbering; Barrels; Social dominance; Grooming; Mutual grooming; Social behavior; Vibrissae; Whisker-trimming

\section{Introduction}

It is commonly observed in laboratory mouse colonies that some cohorts of group-housed mice have vibrissae that are foreshortened or missing and/or have hair missing from the snout, head, shoulders, or forearms. This vibrissae and hair loss has been attributed to the actions of a conspecific, whose actions have been called whisker-trimming, hair-nibbling, whisker-eating, or barbering $[1,2,4,7,9,10]$ or the Dalila effect [12].

The causes of barbering are not known but barbering has been variously attributed to dominance/subordinate status, genetic predisposition, social learning, boredom resulting from poor housing, or dietary deficiencies $[1,2,9,14]$. Long [9] reports that barbering is observed only after the social hierarchy within the cage has been established through aggression, suggesting that it is an

\footnotetext{
* Corresponding author. Tel.: + 1-403-329-2402; fax: + 1-403-3292555 .

E-mail address: whishaw@uleth.ca (I.Q. Whishaw)
}

expression of dominance. Strozik and Festing [14] report that the barber is usually dominant in the 'tube dominance' test (two animals are introduced into opposite ends of a plastic tube and the animal who forces the other one backwards is classified as dominant). Barbering is also more commonly found in certain mouse strains, predominantly the C57BL6 and A2G strains, suggesting that there is a genetic component [7]. Crossfostering experiments, however, suggest that both inheritance and learning contribute to barbering: genetically predisposed pups raised with non-barbering foster parents will still barber, and non-barbering strain pups raised with barbering foster parents will also barber [1]. Van den Broek et al. [15] suggested that barbering may be a form of coping with inappropriate housing. They found that introduced toys reduce barbering, especially if started when the mice were first grouped [2].

Although it appears that barbering involves one active (barber) animal and one passive (recipient) animal, Van Den Broek et al. [15] suggest that it is a co-opera- 
tive. They report that when mice pairs are separated by a wire mesh screen, barbering continues, while when two screens, $10 \mathrm{~mm}$ apart, separate the animals, whiskers regrow. This suggests that the recipient must co-operate with the barber. By directly observing mice barber, Hanschka [7] reported that hair is removed by nibbling, although precisely what 'nibbling' consists of is not described. Whereas subsequent studies have characterized barbering in terms of such end-point measures (presence or absence of whiskers), the primary purpose of the present study was to used videoanalysis to examine how a barber removes its victim's hair. At the completion of the study, measures were made of body and brain weights, and the morphology of cortical barrel-region cells.

\section{Materials and methods}

\subsection{Subjects}

The mouse colony consisted of approximately 100

\section{Barber}

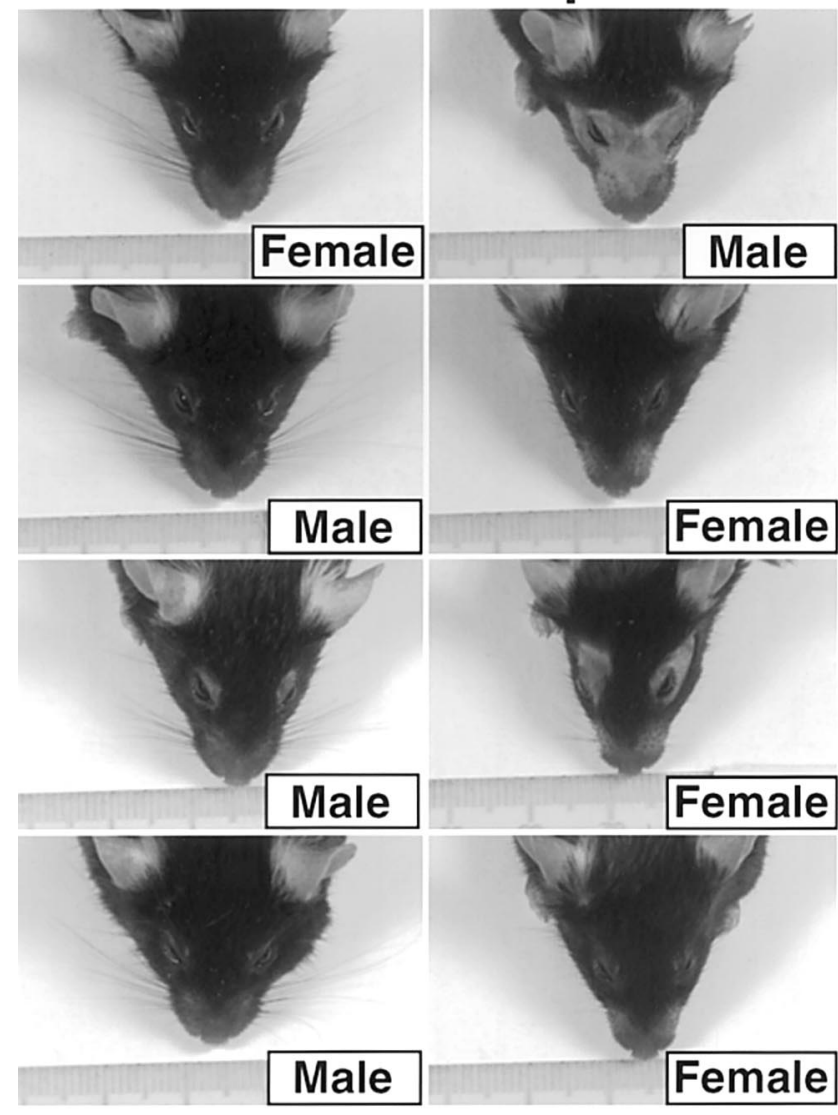

Fig. 1. Appearance of four barbers (left) and four recipients (right). Note that patterns of hair loss differ across the pairs from complete snout denuding (pair 1), vibrissae removal (pair 2), vibrissae and periorbital hair removal (pair 3). and shout vibrissae and hair removal (pair 4).
C57BL6 mice. Among these, 15 mice were found with extensive loss of vibrissae and/or hair. Among these animals, 12 mice were in breeding pairs (three male barber and three female barber pairs) and 12 were from same-sex cages, in which one animal was intact and the remaining had vibrissae and/or hair missing. The hair loss patterns of these animals were observed during the course of the study, and at the completion of the study, the animals were weighed, sacrificed, and their brains removed and weighed. The brains of the animals were subjected to further histological procedures, not reported here.

Seven pairs of animals were chosen for the behavioral studies (control pairs, $n=3$; barbering pairs, $n=$ 4). Typical patterns of hair loss in barber and recipient animals in this group are shown in Fig. 1. Note that one member of each pair has much more striking hair loss than its pair mate. The mice were housed in pairs or singly, depending upon the experimental conditions, in plastic cages lined with wood shavings, in an animal colony lighted on a $12 \mathrm{~h}: 12 \mathrm{~h}$ light-dark cycle, lights off at 20:00 h. Food chow and water was available ad libitum.

\subsection{Filming cylinder for 1 -h test}

The cylinder used for filming of spontaneous activity in the 1-h test was made of clear Plexiglas and measured $40 \mathrm{~cm}$ in diameter and $45 \mathrm{~cm}$ in height. The floor was covered with wood shavings.

\subsection{Video-recording and analysis}

Videorecordings were made using a Canon ES980 videocamera with a shutter speed of 1000 per second. The videotapes were analyzed frame-by-frame (30 frames/s) using a Sony EV-S900 tape deck. Individual behavioral acts were recorded by depressing keys that activated a microcomputer which summarized behavioral acts and their duration. Pictures were obtained using a frame grabber and manipulated on the computer utilizing PHOTOSHOP and CANVAS software packages.

\section{Procedure}

\subsection{One-hour test after separation}

Control $(n=3)$ and barbering $(n=4)$ mouse breeding pairs were separated for a period of 4 weeks. Barbering pairs were those in which one member had its whiskers and/or hair missing, while control pairs were those in which both members were intact. At the end of this period, pairs were reintroduced into a novel Plexiglas 


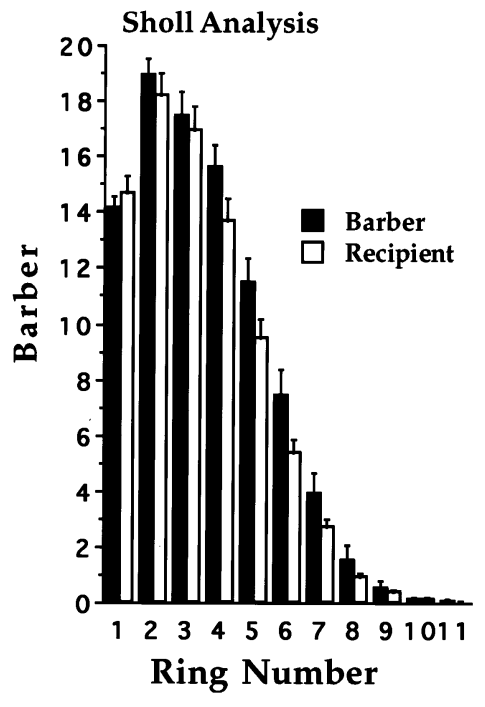

Fig. 2. Scholl analysis of layer three basilar dendrites.

cylinder, and the behavior of the mice was filmed from above for $1 \mathrm{~h}$. The behavior was then analyzed from the video. Ethograms were generated in which each behavior was coded by one letter for both male and female animals. The interactions between the two were boxed. The observed behaviors are were: s, mutual snout sniff; $\mathrm{x}$, general activity including walking, touching the wall of the cylinder and digging; $p$, pursuit of the other animal; a, anogenital sniff; c, climbing onto the back of the other animal; g, grooming; b, soliciting (laying flat by the cage-mate); $\mathrm{m}$, mounting from the rear or side; $f$, frontal mount (mounting the snout of the other animal); $\mathrm{G}$, mutual grooming; $\mathrm{D}$, de-whiskering. The frequency of the behaviors was obtained from the ethograms and analyzed statistically, and, in addition, time spent on grooming, mutual grooming and de-whiskering was recorded and also analyzed (analyses of variance and follow-up $t$-tests) [19].

\subsection{Histological methods}

At the completion of the experiments, the animals were weighed, perfused under deep anesthesia, and their brains harvested and weighted. The brains were placed in a Golgi-Cox solution for 14 days before being sectioned at $200 \mu \mathrm{m}$ on a vibratome and then processed for Golgi-Cox staining [5]. Five pyramidal neurons from layer three of the Zilles parietal barrel field area were selected and their basal dendrites drawn for each hemisphere of each rat [6]. Branch length was determined using the method of Sholl [13]. Statistical analyses were performed by averaging across all cells per hemisphere. Since many mice are barbered from infancy, the brains from separate groups of animals were subject to staining for cytochrome oxidize ([11]; $n=6$ control and $n=6$ barber mice) and zinc staining ([3]; $n=6$ control and $n=6$ barber mice).

\section{Results}

\subsection{Histological results}

Body and brain weights were analyzed with sex and barber status as independent variables. For body weight, there were significant sex differences $(F(1,20)=9.43, P=0.006)$ and barber versus recipient differences $(F(1,20)=9.03, P=0.007)$, but the sex by status interaction was not significant $(F(1,20)=2.66$, $P=0.10)$. Group comparisons showed that the male barbers were significantly larger than their female recipients, whereas female barbers were approximately the same size as their recipients. There were no significant differences in brain weights.

Histological analysis of the barrel fields of control and barbered mice using cytochrome oxidize stains and zinc stains indicated no obvious differences in the organization of the barrels and their size. Intersubject stain variability precluded densitometer comparisons. A Scholl analysis of basilar dendrites, although not giving a difference between barber and recipients $(F(1,20)=$ 2.37, $P=0.13)$, did give a sex difference $(F(1,20)=$ 5.66, $P=0.02)$ and a ring by barber-recipient status difference $(F(12,240)=2.0, \quad P<0.02)$. Overall, dendritic density was larger in female mice, lower in both the male and female recipients, and the reduction in dendritic density in the recipient mice was greatest in rings $3-9$ (Fig. 2).

\subsection{One hour test after separation}

After four weeks of separation, the vibrissae and hair of all barbered animals had completely regrown. After two weeks of reunion, all of the original barbers had mostly intact vibrissae and fur, while all of the recipients had suffered various degrees of vibrissae and hair loss. The pattern of vibrissae and hair loss originally observed in the recipients was closely matched after reunion.

A frame-by-frame analysis of the control and barberrecipient pairs in a 1-h test of social behavior revealed that the animals were both active and interactive (Fig. 3). Comparisons of the incidence of 11 behaviors displayed by control, barber, and recipient animals gave two significant group differences: mounting and barbering. The control pairs displayed more instances of mounting while barbers in the barber-recipient pairs were the only animals to display barbering. These differences are illustrated in the activity ethogram for a control and barber-recipient pair (Fig. 4). 


\subsection{Barbering strategies}

In all observations of barbering in the 1-h test, the barber was as likely to approach as to be approached by a recipient, just prior to an instant of barbering. Nevertheless, in all encounters, the recipient adopted a subordinate and immobile posture, while the barber mounted, manipulated, and barbered. Fig. 5 illustrates typical postures adopted by the barber and the recipient. In most instances, the recipient's eyes were closed and ears were pulled back, and it remained in a prone posture. The barber frequently used one or both of its paws to hold the recipient in place or restrained its movement by laying on top of it.

A typical act of barbering is illustrated in Fig. 6, in which the barber grasps a single vibrissae between its incisors and then pulls, usually pulling the vibrissae out by its roots. All instances of barbering featured grasping and pulling vibrissae with the incisors. Once having pulled a vibrissae from the snout of the recipient, the barber frequently sat back on its haunches, transferred the vibrissae to its paws and proceeded to chew on it.

\section{Discussion}

The present study describes how mice barber , and examines the effect of barbering on morphological factors of body size, brain weight, and somatosensory barrel fields. In a colony of C57BL6 mice, certain individuals in cage cohorts were more likely to be barbers, whereas others were more likely to be recipients. An examination of the barbered animals indicated that the pattern of vibrissae and hair loss in each animal differed to a surprising degree, with some ani-

\begin{tabular}{lllll} 
Behaviour & $\begin{array}{l}\text { Control } \\
n=6\end{array}$ & $\begin{array}{l}\text { Recipient } \\
n=4\end{array}$ & $\begin{array}{l}\text { Barber } \\
n=4\end{array}$ & p-value \\
\hline Mutual Sniffing & 92.33 & 58.50 & 58.50 & $\mathbf{0 . 1 0 4 0}$ \\
Anogenital Sniffing & 60.83 & 33.75 & 49.00 & $\mathbf{0 . 6 1 6 9}$ \\
General Activity & 54.67 & 64.75 & 69.25 & $\mathbf{0 . 2 4 5 7}$ \\
Pursue & 34.33 & 12.50 & 27.50 & $\mathbf{0 . 3 7 0 0}$ \\
Climbing & 23.50 & 1.0 & 11.75 & $\mathbf{0 . 4 6 9 2}$ \\
Soliciting & 3.00 & 8.50 & 19.00 & $\mathbf{0 . 5 0 2 2}$ \\
Frontal Mounting & 13.67 & 0.500 & 0.500 & $\mathbf{0 . 0 6 8 1}$ \\
Mounting & 76.33 & 11.00 & 11.00 & $\mathbf{0 . 0 2 0 6}$ * \\
Grooming & 6.33 & 9.75 & 7.75 & $\mathbf{0 . 4 3 3 7}$ \\
Mutual Grooming & 0 & 4.75 & 2.50 & $\mathbf{0 . 2 9 7 5}$ \\
De-whiskering & 0 & 0 & 7 & $\mathbf{0 . 0 3 3 6}$ \\
\hline
\end{tabular}

Fig. 3. Mean frequency of behaviors in the 1-h filming test. Control animals only differ from the barbering animals in the frequency of mounting behavior (higher in control), mutual grooming and dewhiskering (both absent in control animals). mals displaying only a loss of vibrissae, whereas others had a loss of vibrissae and facial hair. Even the pattern of facial hair loss varied from animal to animal, with some animals displaying hair loss only on the snout, whereas other animals had hair loss on other parts of the face and on the body. Pairs of animals, in which one was a barber and the other was a recipient, were separated in order to allow the hair of the barbered animals to regrow. Surprisingly, the pattern of barbered hair following a period of reassociation again resembled the pattern of hair loss prior to separation. This result suggests that barbers have 'cutting styles'. It would interesting to examine this phenomenon further by examining whether a mouse would barber a number of different animals in the same way.

When the separated animals were re-paired for a session of filming, they engaged in a variety of social interactions, including barbering. An analysis of the videorecording indicated that the recipient was just as likely to approach the barber as the barber was to approach the recipient. In large number of barbering interactions, the barbered animal remained prone and passive while the barber worked. The videorecording indicated that in all cases whisker removal was achieved by grasping a whisker with the incisors and plucking. This appeared 'painful' because the recipient winced. Nevertheless, in no cases did the recipient attempt to escape. Our results are in agreement with van den Broek et al. [15], who claimed that recipients choose to be barbered. Although the recipients presumably experience pain, there could be two motivational forces at work. Perhaps the recipient passively permits barbering to occur because it represses aggression from the barber or it finds the activity enjoyable. In fact, van den Broek et al. [15] speculates that if endorphins are released by barbering, both animals may find the act pleasurable.

The incident of barbering in our colony (circa 20\%) approximates that reported by the $17 \%$ reported in C57BL6 mice by Long [9]. Our study was not directed to the question of why one animals should be a barber and another a victim, but our results do seem consistent with previous work that suggests that the barber is the dominant animal [9]. That is, our observations showed that the recipient sought out the barber, was passive during the barbering procedure, and did not attempt to escape despite the fact that hair plucking must have been painful. The barbers were also the heavier animals, especially the male barbers.

Since all animals do not barber, it is of some interest to question why some animals barber and others do not. It is interesting that the ethogram of the activity of reunited cage mates showed that barber/recipient pairs engaged in both mutual grooming and barbering. We can speculate that barbering may arise in some way as 


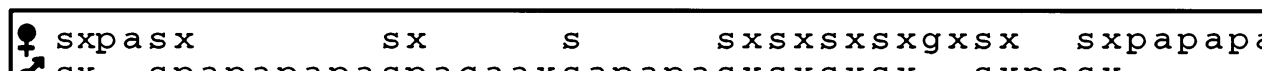
of sx spapapapaspacaaxsapapasxsxsxs sxpasx

$s$ bsapas $s$ s m smx s xsxs sx m xs

sa sx spapacpasascamasmapaacsa sxspasxpamc saxa

ri. sms mssm $m x$ mxm $x$ apaspas msbpaxs $m x m x$ mpaamscmssmam pamxmaxa s sapams xscamxamgp

nsms $m$ ms $m$ mxapapasms mxsmx mxs mx msxsx $m m x$ fmsmsampmsampm smsamxsmxam spm fmsxsxamamxg

spaspasmxsmsmbx $m$ msmx mxasmx $m$ xbsx mxa $m x m$ $s \quad s \quad s m x s m s m$ xpmpmsmxpma smxpmca sxpmx pamxcma msm saspapasx msm xasmx ms $m m x$ s $m$ sxs msx $m$ rsmas $s$ sxgpmsmax sm xamspmpm xsamasxspmsxpma $x \quad m x \quad s m a c p a p a p a c x$ mamxs mg msmxm mxsm $m$ smx $m$ pfm gpsmx am $m$ spmgpmsmpmamxsmamcsm gpm

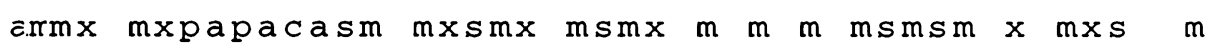
$\mathrm{mm}$ pm smam sm amsmxpmamamcmsmsmapam spamf

rxs $m$ mcpa ps maxax msmmxcsmxs mms mxsmapacsx r. $s a m p m$ gam xpmsmm smxsammsapm sm sxpa

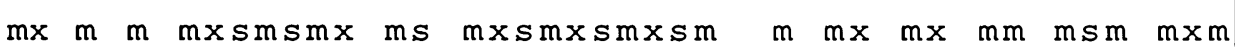
m pmamam smsmxpmsamxsmxsm smpamam gm ammamsmamxm

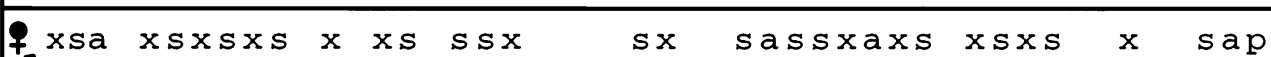
o xsaaxsxixsaxpxsassxaapasxaxs ssx scxsxspaxpas

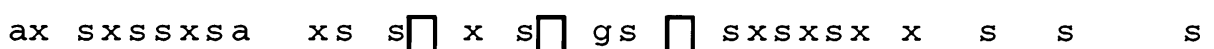
x gsxssxica sasDcxpsDa scDxsxiscaxapscasapaasc

x psbpssxg s

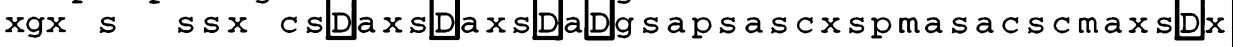

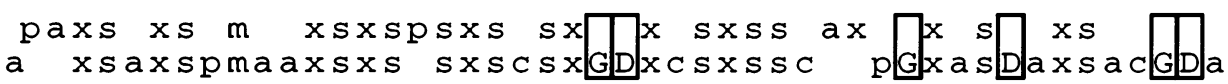

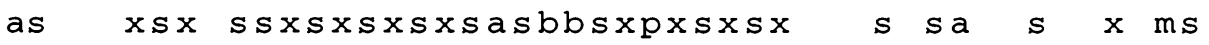
sxagxsxassxsxisxis xsx sxsxaxpsxs xasacxamsa

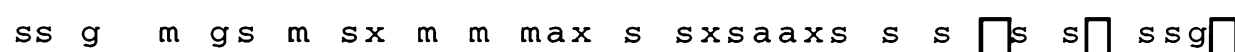
ssaxpama samasxamamamx asasxs xsasascDsasDass D

99 mspa xs $m x \mathrm{~m} m$ mxaxassxsxg msx sxs D] Dlpms axsamxamamamx $x$ ssxsx amsxaxsxs

Fig. 4. Representative ethograms of a control and a barbering pair in a 1-h test (top line, female; bottom line, male). Boxes show interactions: dark boxes emphasize grooming and barbering interactions. Note that control animals do not display de-whiskering or mutual grooming behaviors. s, Mutual snout sniff; $x$, general activity including righting, touching the wall of the cylinder and digging; $p$, pursuit of the other animal; a, anogenital sniff; c, climbing onto the back of the other animal; g, grooming; b, soliciting; m, mating; f, frontal mount; G, mutual grooming; $\mathrm{D}$, de-whiskering).

a product of mutual grooming. We also observed that many mice sat back on their haunches, held a plucked vibrissae in the forepaws and then proceed to eat it in the way that they might eat a piece of food [17]. Since mice typically pick up food with their incisors before transferring it to their paws [18], barbering could begin as an attempt to eat. Some barbered mice do have vibrissae that appear to have been cut and it is possible that the barber may have cut the vibrissae in an attempt to eat it, or while pulling on it. We did not observe such behavior, however, and foreshortened whiskers could also be regrowing whiskers. Finally, we observed that there were more instances of mounting in control mouse pairs. Since mounting is an expression of domi- 
nance [10], it is possible that barbering occurs in mouse pairs in which normal dominance relationships have not formed.

Evidence was obtained for morphological consequences for being a recipient for barbering. In male mice, body weight was reduced, which no doubt reflects social status, but brain size was not affected. Cytochrome oxidize and zinc stains of recipients indicated the presence of barrel fields, although variability in staining precluded group comparisons using densitometer measures. An analysis of the morphology of basilar dendrites from layer three, barrel-field cells indicated there was a reduction in dendritic density in recipient mice, especially in the outer portions of the dendrites. This result indicates that barbering does have conse- quences for brain organization and function, as does artificial whisker trimming $[8,16]$.

In conclusion, mice are becoming a favorite species used for the neural and genetic analysis of behavior. Barbering is an interesting behavior that, although aberrant, may signify the social status of individual animals. Our observation that barbering involves hair plucking also raises the interesting question of why the recipient submits to a 'painful' act. Barbering also appears to have consequences for brain function in recipients as there were morphological changes in barrel field dendrites. Finally, grasping a single hair or vibrissae with the incisors involves considerable oral skill and manipulative skill, and so may be used as a sign that an animal is motorically competent.

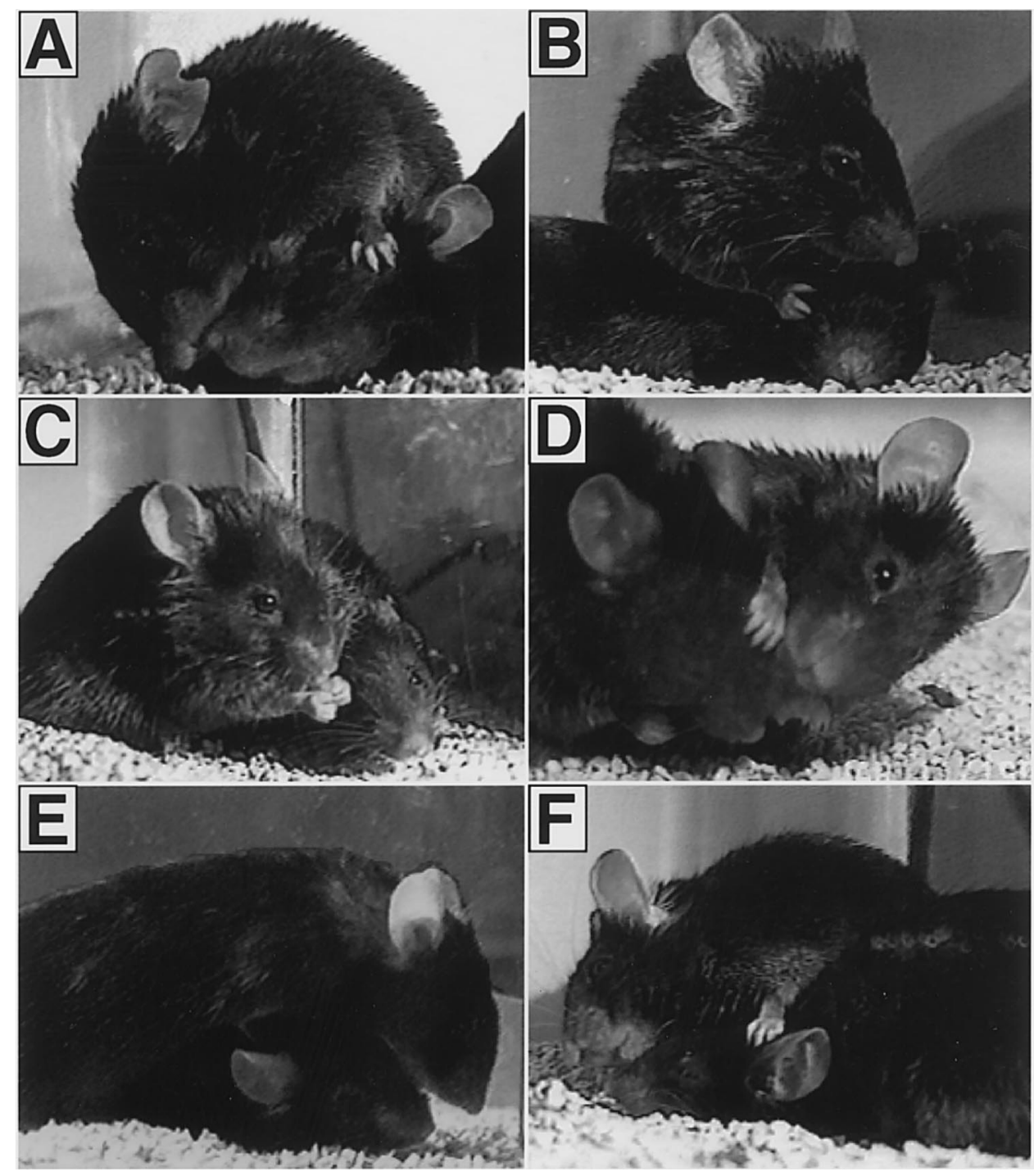

Fig. 5. Barbering postures. (A) The barber is partially on top of the recipient, holding it down with one paw while attempting to grip a whisker with its incisors. (B) The barber holds down the recipient while locating the hair around eyes for removal. (C) The barber consuming a removed whisker. Note that the recipient continues to lay flat in a subordinate position even though the barber is not restraining it physically. (D) The barber holds the snout of the recipient. (E) The barber is laying on top of the recipient while pulling out a whisker. (F) The barber is partially restricting the movement of the recipient while pulling out a whisker by placing one paw and most of its body weight on the head of the recipient. Note that in all instances the recipient has is prone and has its ears back and eyes partially or entirely closed, indicating a subordinate posture. 


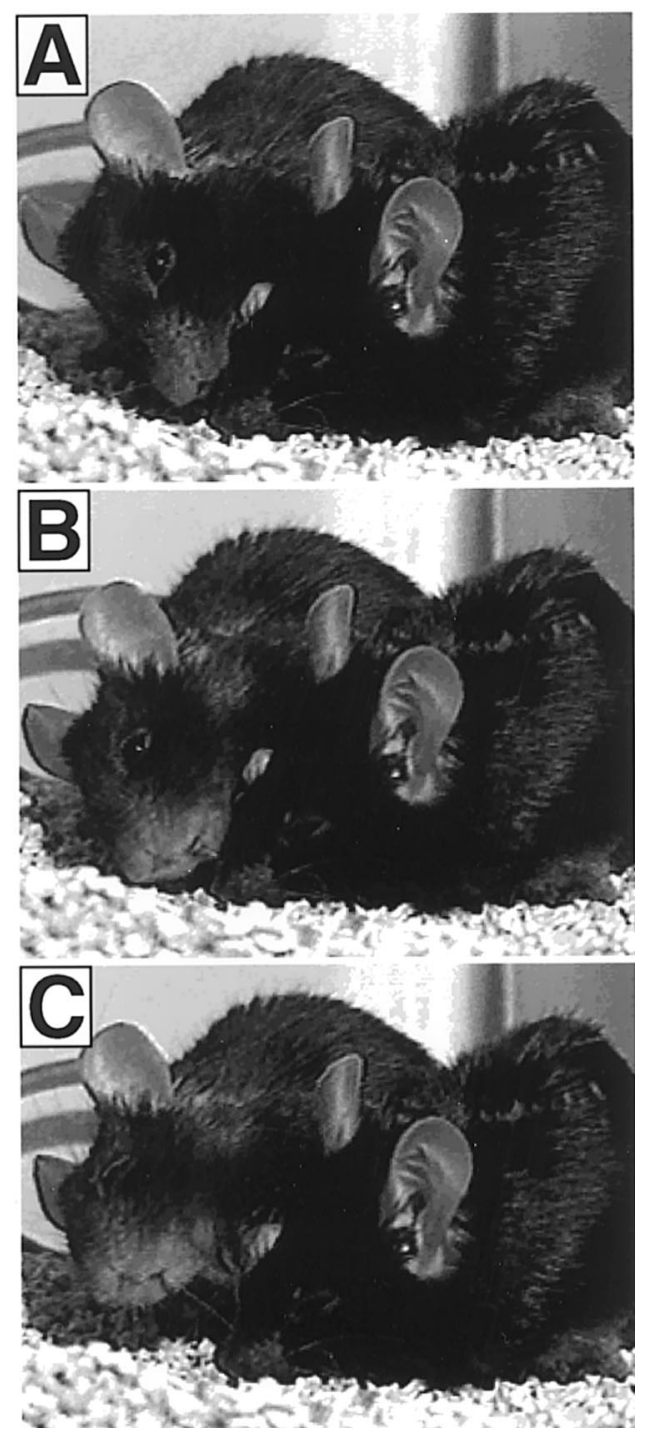

Fig. 6. A typical barbering act. (A) The barber is holding the immobile recipient while it grasps a vibrissae. (B) The barber holds the vibrissae in its incisors and pulls. (C) A hard tug pulls the vibrissae out by the roots.

\section{Acknowledgements}

J.R.S. was supported by a grant from AHFMR.

\section{References}

[1] Carruthers EL, Halkin SL, King TR. Mouse barbering: investigations of genetic and experiential control. Anim Behav Soc Abstr 1998;P41.

[2] DeLuca AM. Environmental enrichment: does it reduce barbering in mice? Anim Welfare Inf Center Newslett 1997;8:7-8.

[3] Dyck RH, O'Kusky JR. Increased cytochrome oxidase activity of mesencephalic neurons in developing rats displaying methylmercury-induced movement and postural disorders. Neurosci Lett 1988;89:271-6.

[4] Ehrenlechner S, Unshelm J. Whisker trimming by mother cats. Appl Anim Behav Sci 1997;52:281-5.

[5] Gibb R, Kolb B. A method for vibratome sectioning of GolgiCox stained whole rat brain. J Neurosci Method 1998;79:1-4 unpublished manuscript, 1992.

[6] Gottlieb JP, Keller A. Intrinsic circuitry and physiological properties of pyramidal neurons in rat barrel cortex. Exp Brain Res 1997;115:47-60.

[7] Hanchska TS. Wisker-eating mice. J Hered 1952;77-80.

[8] Kuljis RO. Vibrissaeless mutant rats with a modular representation of innervated sinus hair follicles in the cerebral cortex. Exp Neurol 1992;115:146-50.

[9] Long S. Hair-nibbling and whisker-trimming as indicators of social hierarchy in mice. Anim Behav 1972;20:10-2.

[10] Pellis SM, Pellis VC. Play fighting of rats in comparative perspective: a schema for neurobehavioral. Neurosci Biobehav Rev 1998;23:87-101.

[11] Quaye VL, Shammalla-Hannah L, Land PW. Experience-dependent alteration of zinc-containing circuits in somatosensory cortex of the mouse. Brain Res Dev Brain Res 1999;114:283-7.

[12] Sarna JR, Dyck RH, Whishaw IQ. The Dalila effect. Paper presented at the Alberta Motor Control Meeting, Jasper, Alta, 1998.

[13] Sholl DA. The Organization of the Cerebral Cortex. London: Methuen, 1956.

[14] Strozik E, Festing MFW. Whisker trimming in mice. Lab Anim 1981;15:309-12.

[15] Van den Broek FAR, Omtzigt CM, Beynen AC. Whisker trimming behaviour in $\mathrm{A} 2 \mathrm{G}$ mice is not prevented by offering means of withdrawal from it. Lab Anim 1993;27:270-2.

[16] Vees AM, Micheva KD, Beaulieu C, Descarries L. Increased number and size of dendritic spines in ipsilateral barrel field cortex following unilateral whisker trimming in postnatal rat. J Comp Neurol 1998;400:110-24.

[17] Whishaw IQ, Coles B. Varieties of paw and digit movement during spontaneous food handling in rats: postures, bimanual coordination, preferences, and effect of forelimb cortex lesions. Behav Brain Res 1996;77:135-48.

[18] Whishaw IQ, Sarna RJ, Pellis SM. Evidence for rodent-common and species-typical limb and digit use in eating, derived from a comparative analysis of ten rodent species. Behav Brain Res 1998;96:79-91.

[19] Winer BJ. Statistical Principles in Experimental Design. New York: McGraw-Hill, 1962. 